\title{
Reflexiones sobre el impacto de la formación en la empleabilidad ${ }^{1}$
}

\author{
Francisco António Vaz Guedes Delgado Ferraz \\ francisco.ferraz@ipleiria.pt \\ Centro de Investigação em Gestão para a Sustentabilidade \\ Escola Superior de Tecnologia e Gestão \\ Instituto Politécnico de Leiria \\ Doutor em Economia Financeira e Contabilidade \\ Equiparado a Assistente do $1 .^{\circ}$ Triénio \\ Morro do Lena, Alto do Vieiro \\ P-2411 - 901 Leiria, Portugal \\ Telef.: 244 820300; Fax: 244820310 \\ Dolores Gallardo-Vázquez \\ dgallard@unex.es \\ Profesora Titular de Universidad \\ Departamento de Economía Financiera y Contabilidad \\ Facultad de Ciencias Económicas y Empresariales \\ Universidad de Extremadura \\ Av. de Elvas $s / n$ \\ 06006 Badajoz (ESPAÑA) \\ Tfno: 924 289520, ext. 89163; Fax: 924272509
}

\begin{abstract}
Resumen
En el mundo actual, la importancia de obtener un desarrollo continuo y equilibrado, basado en un crecimiento generalizado tripartido, inteligente, sostenible e inclusivo se impone cada vez más como una necesidad global permitiendo enfrentar un conjunto de problemas y dificultades causadas por diversos factores tanto ambientales, económicos como sociales. En este contexto las estrategias que permitan impulsar este crecimiento son relevantes, determinando el establecimiento de metas generalizadas y objetivos concretos a alcanzar. Entre las mismas, se destaca el papel de distintos instrumentos, en particular la formación, al servicio de las organizaciones colaborando en la valoración de los recursos humanos y en su inclusión en las mismas. El presente estudio tiene como objetivo reflexionar sobre el efecto de la formación en la empleabilidad de las organizaciones, partiendo de un primer acercamiento teórico al tema y continuando con algunas experiencias en empresas portuguesas acerca del impacto de las prácticas de formación de sus empleados. Las conclusiones permiten relevar una percepción generalizada de la importancia de las prácticas de formación y su influencia en los trabajadores a través de una doble influencia sobre su empleabilidad, fomentando, por un lado, un efecto en su retención en las organizaciones y por otro lado el efecto contrario de fomento de su movilidad.
\end{abstract}

Palavras chave: Empleabilidad, Formación, Estrategia, Recursos Humanos, Inclusión Social, Mercado de Trabajo.

\section{Introducción}

El mundo actual se enfrenta a muchos problemas y dificultades causadas por un conjunto de factores ambientales, económicos y sociales, determinando un permanente estado de transformación. En este contexto, la importancia de obtener un desarrollo continuo y sostenible,

${ }^{1}$ El presente trabajo ha sido financiado con las Ayudas a Grupos de Investigación GR10041, recibidas durante el año 2013 dentro del IV Plan de Actuación 2010-2014 
basado en un crecimiento generalizado se impone cada vez más como una necesidad global, y es importante el papel que los diferentes actores de la actividad económica y la sociedad tienen, y en particular las empresas. En este contexto, sus acciones deben tratar de responder a una serie de nuevas necesidades y realidades diferentes mediante la obtención de un conjunto de respuestas eficientes y eficaces a estos retos, y por lo tanto determinando la existencia de estrategias y directrices que permitan establecer metas generalizadas y objetivos concretos a alcanzar.

Una de las estrategias formuladas por la Comisión Europea, la Estrategia UE 2020 (COM, 2010), pretende reforzar la importancia y el favorecimiento de este crecimiento, tripartito en tres dimensiones, inteligencia, sostenibilidad y de inclusión. En este trinomio, destacamos el papel de distintos instrumentos, en particular la formación, al servicio de las organizaciones colaborando en la inclusión de los empleados de las mismas. Teniendo en cuenta estas consideraciones, la cuestión de la valoración de los recursos humanos se está convirtiendo cada vez más en una importante herramienta que influye en el aumento de las capacidades de los trabajadores, así como en el valor para la organización. Además, la importancia de los intangibles surge asociada al crecimiento de las organizaciones, en la medida que posibilita el desarrollo de condiciones ante la adaptación a nuevas exigencias sociales y laborales. Por lo tanto, es importante la existencia de estudios y reflexiones sobre el papel que la formación tiene en el refuerzo del conocimiento de los trabajadores y su impacto en la relación de éstos con la organización, buscando este documento, a través de una base de análisis descriptiva en un conjunto de diversas opiniones, informes, artículos e investigaciones en el tema, contribuir a este hecho.

El artículo se estructura en cuatro partes. En un primer momento, se presenta el tema de la empleabilidad, después su relación con el desarrollo sostenible y en una tercera parte la formación y sus efectos en las organizaciones. Finalmente, se presentan las conclusiones más significativas y se exponen algunas propuestas para futuras investigaciones.

\section{Empleabilidad}

La empleabilidad presenta cada vez más un papel destacado en el crecimiento de las sociedades como factor de integración de los recursos humanos en las organizaciones y de inclusión social. El término tiene un origen anglo-sajón en el inicio del siglo XX, siendo utilizado para distinguir la población empleable y no empleable (Almeida, 2007) y su difusión internacional se ha 
realizado a través de la contribución relevante de un conjunto de diversas organizaciones internacionales ${ }^{2}$.

Su concepto puede ser analizado bajo diferentes perspectivas, estando siempre presente la figura del trabajador, es decir, el individuo y también del mercado de trabajo. También es fundamental el factor de unión o de interactividad entre estos dos componentes o partes. Se presupone así una interacción entre las partes distintas, las características personales y el mercado de trabajo, es decir, las estrategias y los recursos individuales, por un lado, las dinámicas macroeconómicas y las estrategias empresariales, por otro (Gazier, 1999; Chiavenato, 2006). En este proceso, son importantes determinados aspectos, como el referido por Mcquaid y Lindsay (2005), relacionado con el papel del individuo así como su relación con el mercado de trabajo. En relación con el individuo, deben de ser considerados factores relacionados con circunstancias internas o personales, y en relación con el mercado de trabajo deben de ser considerados factores que son externos al trabajador.

En relación a los factores individuales están asociados a las competencias y atributos que influyen en la empleabilidad, que incluyen los grados escolares y cualificaciones profesionales de los individuos así como un conjunto de competencias de naturaleza diversa que pasan, entre otras, por sus competencias sociales, comportamentales, de resolución de problemas y de la adaptación a nuevas situaciones. Las contribuciones de las teorías del capital humano están patentes en este concepto de empleabilidad a través de la valoración del papel de la formación. Este aspecto se relaciona con las circunstancias personales que son afectadas por los recursos y que permitirán el acceso al consumo (capital financiero) o que permiten el acceso a la información y grupos de status (capital social).

En relación a los factores externos al trabajador, los mismos están asociados a las dinámicas del mercado, tendencias macroeconómicas, formas contractuales, a las condiciones del trabajo y a las políticas de reclutamiento de las empresas. Estos son importantes en la medida que impulsan una competitividad dinámica a través de la capacidad de innovación, el nivel de los procesos productivos, de los productos y de la base organizacional (Rodrigues, 1998).

\footnotetext{
${ }^{2}$ Organización Internacional del Trabajo (OIT), la Organización para la Cooperación y Desarrollo Económico (OCDE), la Organización de las Naciones Unidas (ONU) y la Unión Europea (UE).
} 
Las empresas en este proceso tienen un papel importante, como es indicado por Almeida (2007), pues la construcción de la empleabilidad pasa por un proceso que tiene diversas prácticas y procesos concretamente de gestión de los recursos humanos en la medida que exige un aprendizaje de cambio, siendo estas prácticas capaces de apoyar procesos de innovación técnica y organizacional indispensables ante la competitividad moderna. La intervención de las empresas, permite promover procesos de aprendizaje y por lo tanto éstas tienen algunos mecanismos disponibles, entre ellos las políticas de formación. Al estructurar y adoptar políticas coherentes y estructuradas de formación profesional, pueden las organizaciones reforzar su competitividad así como implicar y comprometer a los empleados de la organización con ésta, a través de la respectiva valoración de sus competencias.

En un ambiente de hipercompetitividad en que las empresas desarrollan sus actividades existen diversas dificultades y apenas conseguirán sobrevivir las más hábiles y capaces de prevenir los cambios, a través de la creación de ventajas competitivas continuas, como señala Alves (2007). Este autor indica que esa ventaja auto-sustentada serán los activos humanos, a través no sólo de sus recursos físicos sino también mentales y emocionales. Este autor desarrolla un concepto de organizaciones de aprendizaje, que considera a las personas como factor crítico del éxito.

En este contexto, diversos autores señalan la relación del desarrollo personal de los trabajadores a través de la formación con la empleabilidad (Maclean y Ordonez, 2007; Bulut y Culha, 2010; Collier et al., 2011). Así, este concepto es entonces un proceso apoyado por un aprendizaje permanente orientado al largo plazo, apostando en el capital humano y en competencias.

\section{La Empleabilidad y el Desarrollo Sostenible}

La preocupación por un desarrollo en general, continuo, a largo plazo, forma parte de los objetivos mundiales tanto de las sociedades como de las organizaciones y comunidades globales. Hay una necesidad global por la obtención de un diferente tipo de desarrollo, que haga frente a los desafíos ambientales, económicos y sociales, motivados por el estado de permanente transformación e inestabilidad. En este contexto, el desarrollo sostenible es un objetivo mundial, como es referido por la Comisión de las Comunidades Europeas (COM, 2006).

Su obtención corresponde a un desafío que no podrá ser alcanzado si no hay un empeño global de los países, ciudadanos y organizaciones. Así deberá existir una contribución concertada y cohesiva por parte de todos estos intervinientes, siendo importante la existencia de estrategias 
que lo permitan. De acuerdo con la estrategia propuesta por la Comisión de las Comunidades Europeas (COM, 2001), denominada "Estrategia de la Unión Europea en favor del desarrollo sostenible", o más conocida por "Estrategia Europea de Desarrollo Sostenible", hay una preocupación generalizada por el conjunto de problemas que pueden amenazar el bienestar de la sociedad europea, tales como el calentamiento global, las múltiples variedades resistentes a los antibióticos de ciertas enfermedades y amenazas a la seguridad de los productos alimenticios, la pobreza, el envejecimiento de la población, la pérdida de la diversidad biológica así como los niveles de residuos o el exceso de los transportes y desequilibrios regionales.

Para corregir todas estas tendencias que eviten una sostenibilidad es necesario "una acción urgente, liderazgo político comprometido y perspicaz, un nuevo enfoque en la toma de decisiones políticas y una amplia participación y responsabilidad internacional" (Comisión de las Comunidades Europeas, 2001:4). Así, la referida estrategia se apoya básicamente en tres componentes para cumplir con estos objetivos, es decir: Propuestas y recomendaciones transversales que permitan mejorar la eficacia política llevando a que haya un refuerzo mutuo de las diversas políticas; la definición de objetivos principales y medidas específicas que enfrentasen mayores desafíos al desarrollo sostenible y medidas de aplicación de la estrategia y evaluación de sus progresos.

La Comisión ha presentado un conjunto de propuestas base para garantizar respuestas eficaces a los desafíos lanzados, tales como la mejoría de la coherencia política, invertir en la ciencia y en la tecnología para el futuro, mejorar la comunicación y movilizar a los ciudadanos y las empresas así como tomar en consideración la ampliación y la dimensión global.

La estrategia enunciada establece determinados objetivos y finalidades a largo plazo, identificando prioridades de acción, de entre las cuales se destacan la inclusión de los objetivos de la estrategia de Lisboa ocurrida en 2000 en el dominio de la política social tal como el combate a la pobreza y a la exclusión social. Estos objetivos van en la línea de los fines generales definidos en la estrategia de Lisboa de 2000, es decir de hacer de la Unión Europea, hasta 2010, la economía basada en el conocimiento más competitiva del mundo, capaz de garantizar un crecimiento económico sostenible, con más y mejores empleos, y con mayor cohesión social y respeto por el ambiente, conforme señala el Centro de Información Europea Jacques Delors (2007). 
La preocupación de la Unión Europea con estas acciones es relevante, siendo su reflejo la permanente evaluación de las estrategias adoptadas, en particular la Estrategia Europea para el Desarrollo Sostenible. Así, es posible efectuar el análisis de la situación anualmente, permitiendo detectar los progresos alcanzados, evaluando y adaptando métodos de trabajo, así como efectuando el análisis a medio plazo y permitiendo la adaptación de la estrategia a las alteraciones de las prioridades a largo plazo.

En la continuación de la Estrategia del crecimiento se destaca el relanzamiento de la Estrategia de Lisboa, en Marzo de 2005. Esta fase se centró más en relación a dos prioridades: garantizar un crecimiento más sólido y duradero y la creación de más y mejor empleo, al mismo tiempo que gira en torno a la cohesión social y la sostenibilidad ambiental, implicando así un mayor compromiso por parte de los gobiernos de cada país a través de Planes Nacionales.

La Comisión Europea, con este relanzamiento, fortalece su esfuerzo en el objetivo del desarrollo sostenible pero con la conciencia de que no es posible sin más crecimiento y más empleo (Comisión de las Comunidades Europeas, 2005). Esta estrategia relanzada, releva los ejes fundamentales del conocimiento e innovación como motores de un crecimiento sostenible, el hecho de permitir un espacio atractivo para invertir y trabajar y también el crecimiento y el empleo al servicio de la cohesión social. En estos ejes destacan el conocimiento y el saber a través de la formación a lo largo de la vida y el empleo como factor de inclusión social.

Estos desafíos sólo podrán ser alcanzados si existe un esfuerzo y una acción global por parte de los diversos países y movilizando a todos los ciudadanos y organizaciones. Así, el esfuerzo de la ejecución de la estrategia debe de ser compartido entre los individuos, empresas, sociedad civil y las autoridades locales y regionales. El proceso de implementación de la estrategia llevó a la Comisión y el Consejo Europeo a establecer objetivos de largo plazo y a proceder al asesoramiento de los estados-miembros en la elaboración de Planes Nacionales de Reforma y en la coordinación de la implementación de la Estrategia de Lisboa, adecuando al Gobierno de cada estado miembro la responsabilidad por la implementación de la Estrategia de Lisboa.

Los reflejos concretos de esta Estrategia a nivel nacional se hacen a través de la elaboración de los denominados Planes Nacionales de Reformas (PNR), atendiendo al punto de partida de cada país y las respectivas prioridades nacionales (Centro de Información Europea Jacques Delors, 2007). A nivel interno en Portugal, su PNR, denominado Programa Nacional de la Cualificación 
IV Conferência Investigação e Intervenção em Recursos Humanos - Os Novos Contextos da Gestão de Recursos Humanos

Escola Superior de Ciências Empresariais do Instituto Politécnico de Setúbal

28 e 29 de janeiro de 2013

de las Jefaturas Intermedias (PNACE) (2003) es una respuesta a las líneas de orientación de la Estrategia de Lisboa, constituyendo una referencia de encuadramiento para las diversas políticas gubernamentales, de ámbito macroeconómico, microeconómico y de cualificación y empleo.

Este plan comprende un conjunto de programas, entre los cuales se encuentran el Plan Tecnológico (PT), el Programa de Estabilidad y Crecimiento (PEC) y el Plan Nacional del Empleo (PNE). Engloba también un conjunto de orientaciones del denominado Cuadro de Referencia Estratégica Nacional (QREN), referencial estratégico y de encuadramiento de las intervenciones apoyadas por los fondos estructurales y de cohesión, así como de la denominada Estrategia Nacional de Desarrollo Sostenible (ENDS) (Santos y Silva, 2005), instrumento orientador con el objetivo de hacer de Portugal, en el horizonte de 2015, uno de los países más competitivos de la Unión Europea.

En general, se está dando importancia a la cuestión de la empleabilidad, por su relación con el crecimiento y el desarrollo de los países y economías. Es así importante hacer una reflexión sobre herramientas que impulsen la empleabilidad, como la cualificación de los recursos humanos a través de la formación.

\section{La Formación y sus efectos en las organizaciones}

\section{Concepto de la formación}

El concepto de formación es amplio y se define de muchas y diferentes maneras. Diversos autores señalan que el término "formar" está directamente relacionado con las características del individuo, su carácter, personalidad, sistema de valores y actitudes. También se refiere al proceso de desarrollo y mejora tanto de capacidades como de comportamientos y actitudes. Es entonces un concepto dinámico que permite acercar el empleado al trabajo que ocupa (Barrett y O'Connell, 2001; Casanova, 2003, De Vos et al., 2010). Este concepto en un entorno cada vez más cambiante gana una importancia cada vez mayor por su efecto en la adaptación de los trabajadores.

Un análisis al concepto del término "formación" nos remite a la adquisición de habilidades y conocimientos, importantes y necesarios para el desarrollo de tareas en la actividad que los trabajadores hacen, como se señala en la tabla siguiente (tabla $\left.\mathrm{n}^{\circ} 1\right)$ : 
Tabla n¹: Algunas definiciones sobre el concepto de formación

\begin{tabular}{|c|l|}
\hline Mata et al. (2006) & $\begin{array}{l}\text { El conjunto programado de actuaciones sobre los trabajadores con el fin de } \\
\text { proporcionar, adecuar o desarrollar conocimientos y habilidades que les } \\
\text { permitan mejorar su capacidad. }\end{array}$ \\
\hline $\begin{array}{c}\text { Buckley y Caple } \\
\text { (2007) }\end{array}$ & $\begin{array}{l}\text { Es un esfuerzo planificado y sistemático para modificar o desarrollar los } \\
\text { conocimientos, técnicas y actitudes a través de experiencias de aprendizaje y } \\
\text { lograr la actuación adecuada en una actividad o categoría de actividades, con } \\
\text { el propósito de capacitar a un individuo para que pueda realizar } \\
\text { convenientemente una tarea o trabajo determinado. }\end{array}$ \\
\hline $\begin{array}{c}\text { Winters et al. (2009) } \\
\text { Organización }\end{array}$ & $\begin{array}{l}\text { Conjunto de actividades relacionadas con las tareas actuales de los } \\
\text { competencias profesionales o el desarrollo de las ya existentes. }\end{array}$ \\
\hline $\begin{array}{c}\text { Internacional del } \\
\text { Trabajo (2009) }\end{array}$ & $\begin{array}{l}\text { Proceso de desarrollo de conocimientos, competencias y habilidades en } \\
\text { todas las etapas de la vida de la persona y que promueve su empleabilidad. }\end{array}$ \\
\hline Nguyen et al. (2010) & $\begin{array}{l}\text { Elemento que permite el incremento de conocimiento y competencias } \\
\text { necesarias para las actividades de los trabajadores. }\end{array}$ \\
\hline
\end{tabular}

Se verifica, en general, una opinión por una definición que pasa por relevar su papel en el desarrollo personal y profesional de los trabajadores, y por tanto un factor de calidad de los recursos y de competitividad de la organización. Es transversal a todas las tareas de una profesión o grupo de profesiones en cualquier ramo de actividad económica (Dirección General de Empleo y Formación, 2001).

Cada organización adopta una estrategia global teniendo en cuenta un conjunto de factores económicos y financieros, las nuevas tecnologías, su capacidad de innovación, las características de los productos, la legislación vigente, la cultura corporativa y también nuevas preocupaciones que se presenten (Cornelius et al., 2008). En este contexto la formación se integra, por tanto, en la estrategia de recursos humanos de la organización, ajustándose a su vez la misma en la estrategia global de la empresa (Cardon y Stevens, 2004; Lopes, 2005; Cámara y Castilla, 2005; Weber, 2007; Buller y McEvoy, 2012).

El impacto de la formación en la actividad de la empresa se hace en particular en diversas áreas, como la mejora de la eficiencia de los trabajadores con una mayor frecuencia en acciones de formación profesional obteniendo resultados en términos de producción, que pueden ser mayores cuanto mayor sea la complejidad del proceso; el uso más eficiente de los recursos, tomando mejores decisiones, lo que permite desarrollar de la mejor manera las diversas aplicaciones; una mejor adaptación a los nuevos productos y procesos, dando lugar a una introducción más amplia 
y a la difusión de la tecnología y en la promoción de una mejor preparación para la investigación y el desarrollo, lo cual a su vez genera una mayor productividad.

Los objetivos globales europeos de un crecimiento generalizado en los diversos países de la Unión destacan el proceso de innovación como elemento clave para impulsar una competitividad duradera. Así, la formación hace posible que los trabajadores posean unas ciertas cualificaciones y un conjunto de capacidades que les permitirán desarrollar la innovación. A su vez se obtiene una mayor flexibilidad de los activos para realizar determinadas tareas, influye en una reducción de los costes medios, la optimización del trabajo, una rápida adaptación a los cambios tecnológicos y mejores formas de organización del trabajo estimulando los procesos innovadores en las empresas.

Es relevante la existencia de estrategias de recursos humanos que puedan permitir los efectos enunciados, siendo la formación una herramienta importante para obtener estos objetivos (Danvila y Sastre, 2005; Buller y McEvoy, 2012).

\section{Efectos de la formación en los trabajadores}

En general hay una opinión generalizada de los beneficios asociados a la formación y su fomento en el crecimiento económico, a través de la inclusión social, reducción de las desigualdades, realización personal de los activos promoviendo un mayor bienestar de la población, permitiendo la empleabilidad y la adaptabilidad con los mejores empleos.

En las empresas, la formación es señalada por Mata et al. (2006) como un proceso que permite una adaptación al empleo y de forma continua, facilitando los cambios e innovaciones e incrementando el potencial de la empresa. Así, tiene por objeto eliminar las diferencias entre lo que el empleado puede ofrecer de su experiencia, habilidades y actitudes y lo que se requiere del trabajo que ocupa. En este contexto, hay una influencia en la fidelidad del trabajador al trabajo. Esta puede manifestarse por un aumento de la empleabilidad efectiva de los trabajadores, como ha mencionado la Dirección General de Estudios, Estadística y Planificación (DGEEP, 2007).

El conocimiento tiene un impacto en los recursos humanos de la empresa pasando por el aumento de sus capacidades distintivas, es decir, por sus habilidades a través del conocimiento. La Organización Internacional del Trabajo (OIT, 2008:5) destaca este hecho cuando se refiere a la importancia de las organizaciones para proporcionar nuevas habilidades a sus trabajadores con 
el fin de "retenerlos" en la organización. El efecto de la formación en la retención de los trabajadores tiene otros efectos importantes para la economía y para los respectivos trabajadores, tales como los señalados por la Comisión de las Comunidades Europeas, de la influencia en la reducción de los períodos de desempleo (COM, 2010). Este hecho permite uno de los objetivos de la Comisión Europea, a través de la promoción del llamado crecimiento inclusivo, lo que favorece una economía con niveles altos de empleo.

La característica de la dificultad en el reemplazo en el trabajo es señalada por otros autores (Grant, 1991; Barney, 1991) como importante aspecto porque permite a los recursos existentes en la empresa mantener determinadas características que ayudan en el desarrollo de tareas mejorando su competitividad, y también va a limitar la posibilidad de la imitación del éxito de la organización, convirtiéndose en una autentica ventaja competitiva pues sus competidores no tienen estos recursos y que llegan a adquirirlos.

Todavía, la obtención del conocimiento puede no significar su permanencia en las organizaciones, y el mismo puede estar relacionado con otros efectos en la organización, es decir, con una movilidad profesional.

El concepto de la movilidad profesional o laboral puede ser entendido como la frecuencia con que los trabajadores cambian de empleo. La misma presenta un conjunto de ventajas para las economías nacionales, tales como la circulación de la mano de obra y el equilibrio del mercado de trabajo y, por lo tanto, es importante porque puede ser visto como una manera de luchar frente al desempleo. En un contexto de globalización es importante fomentar esta práctica, ya que la movilidad laboral permite obtener economías con más éxito y dinámicas (Winters et al., 2009).

De acuerdo con la Comisión de las Comunidades Europeas (COM, 2002), la movilidad profesional incluye un conjunto de acciones de promoción del desarrollo de las competencias de los trabajadores. Así, de acuerdo con la Comisión, son fundamentales las estrategias de refuerzo e incentivo a la movilidad laboral junto con las estrategias de aprendizaje a lo largo de la vida con objeto de alcanzar el pleno empleo. En este sentido la adquisición de nuevas competencias y la realización de nuevas experiencias permiten beneficiar no solamente a los propios trabajadores sino también a las propias organizaciones.

Para ser posible esta movilidad tiene que existir una continuidad en la formación y cualificación acompañando las diversas transformaciones que existen en el mercado de trabajo, las cuales 
dependen de diversos aspectos como las nuevas tecnologías, la innovación y también la ciencia (Aguinis y Kraiger, 2009).

Las preocupaciones con la formación deben de ser permanentes existiendo la necesidad de la adaptación de la formación a los requisitos del mercado de trabajo, la promoción del aprendizaje a lo largo de la vida y de las competencias (COM, 2002). En el marco de la Estrategia Europa 2020, se asume la importancia de una sociedad basada en el conocimiento, incluyendo los altos niveles de los índices educativos medios, la promoción del aprendizaje a lo largo de la vida y la movilización de los ciudadanos en la sociedad de la información y el conocimiento.

\section{Conclusiones}

El estudio ha permitido verificar un conjunto de referencias teóricas de la importancia de la formación en el desarrollo de los trabajadores, a través de una influencia en sus habilidades y capacidades. Hemos verificado un conjunto de opiniones en general de efectos que derivan de la percepción de la relevancia de las prácticas de formación y su influencia en los trabajadores.

En relación con la innovación podemos decir que, en un contexto de crisis y de alteraciones constantes afectando a las empresas, existe una coyuntura de empleo vulnerable. Así es importante una adaptación o una conversión de los empleados a nuevas exigencias laborales, pudiendo la formación tener influencia en la valorización o adaptación de competencias y habilidades de los empleados. También la misma podrá ser una oportunidad para expandir horizontes y captar un conjunto de nuevas oportunidades para los trabajadores de las empresas.

Otro aspecto destacado es un doble efecto de la formación en los trabajadores, influyendo sus niveles de empleabilidad, tanto internos como externos. En el contexto referido y de acuerdo con el conjunto de autores referidos, podemos encontrar un conjunto de efectos de la formación en los trabajadores de impacto diversificado en su actividad. Por un lado, un efecto, en la empresa donde trabajan, existiendo una retención de los empleados motivada por una dificultad en el reemplazo de su trabajo limitando la posibilidad de su imitación y la reducción de los períodos de desempleo.

Por otro lado, el efecto contrario mediante el fomento de su movilidad pues la formación puede permitir la adquisición de competencias importantes que permitan oportunidades de adaptación a 
otros entornos, lugares y empresas, contribuyendo al movimiento de la mano de obra y el equilibrio del mercado de trabajo.

\section{Limitaciones y propuestas de investigación futura}

En la realización de nuestro estudio hemos encontrado algunas limitaciones que van a influenciar nuestro análisis y los resultados obtenidos. En concreto, nos referimos a la utilización de una metodología no reactiva de recogida de los datos, basada en el análisis del investigador, es decir, basada en el análisis de percepciones y con un cierto grado de subjetividad. Representa una técnica importante en la investigación de las ciencias sociales y se ha utilizado en otros estudios recientes en relación al tema de la empleabilidad (Maclean y Ordonez, 2007).

La identificación de esta limitación nos permite considerar interesante, a continuación, hacer un estudio empírico sobre el impacto de la formación en actividades ligadas a diferentes sectores de actividad, verificando concretamente la influencia e impacto de las prácticas formativas en la empleabilidad de los trabajadores de las organizaciones.

\section{Bibliografia}

Aguinis, H., y Kraiger, K. (2009). Benefits of Training and Development for Individuals and Teams, Organizations, and Society. The Annual Review of Psychology, 60, pp. 451-474.

Almeida, A. (2007). Empregabilidade, contextos de trabalho e funcionamento do mercado de trabalho em Portugal. Revista de Ciências Sociais da Educação, 2, pp. 51-58.

Alves, N. (2007). E se a melhoria da empregabilidade dos jovens escondesse novas formas de desigualdade social?. Revista de Ciências da Educação, 2, pp. 59-68.

Barney, J. (1991). Firm Resources and Sustained Competitive Advantage. Journal of Management, 17(1), pp. 99120.

Barrett, A. y O’Connell, P. (2001). Does Training Generally Work? The Returns to Incompany Training. Industrial and Labor Relations Review, 54(3), pp. 647-675.

Buckley, R. y Caple, J. (2007). The Theory \& Practice of Training. London, Kogan Page Publishers.

Buller, P. y McEvoy, G. (2012). Strategy, human resource management and performance: Sharpening line of sight. Human Resource Management Review, 22(1), pp. 43-56.

Bulut, C. y Culha, O. (2010). The effects of organizational training on organizational commitment. International Journal of Training and Development, 14(4), pp. 309-322.

Cámara de la Fuente, M. y Castilla Polo, F. (2005). Hacia la construcción de modelos de capital intelectual en industrias tradicionales: El caso de las almazaras jiennenses. Revista de Economía Pública, Social y Cooperativa, 46, pp. 191-211.

Cardon, M. y Stevens, C. (2004). Managing human resources in small organizations: What do we know?. Human Resource Management Review, 14(3), pp. 295-323.

Casanova, F. (2003). Formación profesional, productividad y trabajo decente. Boletín Cinterfor, 153, pp. $29-54$.

Centro de Información Europea Jacques Delors, CIEJD (2007). Crescimento e Emprego - Juntos a trabalhar pela Europa do futuro. Edición CIEJD.

Chiavenato, I. (2006). Administração - Teoria, Processo e Prática. São Paulo, McGraw Hill. 
Collier, W., Green, F., Kim, Y. y Peirson, J. (2011). Education, Training and Economic Performance: Evidence from Establishment Survival Data”. Journal of Labor Research, 32(4), pp. 336-361.

Comisión de las Comunidades Europeas (2001). COM(2001)0264, Desenvolvimento sustentável na Europa para um mundo melhor: Estratégia da União Europeia em favor do desenvolvimento sustentável. Bruxelas.

Comisión de las Comunidades Europeas (2002). COM(2002)72, Comunicação da Comissão relativa ao Plano de Ação da Comissão para as Competências e a Mobilidade. Bruxelas

Comisión de las Comunidades Europeas (2005). COM(2005)24 final, Comunicação da Comissão - Trabalhando juntos para o crescimento e o emprego. Um novo começo para a estratégia de Lisboa. Bruxelas.

Comisión de las Comunidades Europeas (2006). COM(2006)136, Implementação da parceria para o crescimento e o emprego: tornar a Europa um pólo de excelência em termos de responsabilidade social das empresas. Bruxelas.

Comisión de las Comunidades Europeas (2010). COM(2010)2020 final, Europa 2020 - Estratégia para um crescimento inteligente, sustentável e inclusivo. Bruxelas.

Cornelius, N., Todres, M., Janjuha, S., Woods, A. y Wallace, J. (2008). Corporate Social Responsibility and the Social Enterprise. Journal of Business Ethics, 81(2), pp. 355-370.

Danvila, V. y Sastre, M. (2005). Problemas y propuestas de medición de la formacíon en la empresa. Cuadernos de Estudios Empresariales, 15, pp. 27-45.

De Vos, A., Hauw, S. Beatrice, Heijden, B. (2011). Competency development and career success: The mediating role of employability. Journal of Vocational Behavior, 79, pp. 438-447.

Dirección General de Estudios, Estadística y Planificación, DGEEP (2007). Estudo sobre o Retorno da Formação Profissional. Lisboa, Coleção Cogitum.

Dirección General de Empleo y Formación, DGEF (2001). Terminologia de Formação Profissional. Coleção DEF.

Gazier, B. (1999). Assurance chômage, employabilité et marchés transitionnels du travail. Paris.

Grant, R. (1991). The resource-based theory of competitive advantage: implications for strategy formulation. California Management Review, 33, pp. 114-135.

Lopes, A. (2005). As consequências da formação sobre a produtividade: uma análise microeconómica com base no Balanço Social. Coimbra.

Maclean, R. y Ordonez, V. (2007). Work, skills, development for employability and education for sustainable development. Educational Research for Policy and Practice, 6(2), pp. 123-140.

Mata, A., Güenagia, J., Rodríguez, J. (2006). Comportamiento de las empresas respecto a la formación continua de sus directivos. Cuadernos de Gestión, 6(1), pp. 83-98.

Mcquaid, W. y Lindsay, C. (2005). The concept of employability. Urban studies, 42, pp. 197-219.

Nguyen, T., Truong, Q. y Buyens, D. (2010). The relationship between training and firm performance: a literature review. Research and Practice in Human Resource Management, 18(1), pp. 28-45.

Organización Internacional del Trabajo, OIT (2008). Conclusiones sobre las calificaciones para la mejora de la productividad, el crecimiento del empleo y el desarrollo. Disponible en http://www.ilo.org (consultado en 14 de enero).

Organización Internacional del Trabajo, OIT (2009). Relatório sobre tendências mundiais de emprego 2009 Global Employment Trends Report 2009. Disponible en http://www.ilo.org (consultado en 15 de enero).

Rodrigues, M. (1998). Competitividade e recursos humanos. Lisboa, D. Quixote.

Santos, M. y Silva, J. (2005). Desenvolvimento Sustentável e Responsabilidade Empresarial. Oeiras, Editora Celta.

Weber, J. (2007). Business Ethics Training: Insights from Learning Theory. Journal of Business Ethics, 70, pp. 6185 .

Winters, A., Meijers, F., Kuijpers, M. y Baert, H. (2009). What are vocational training conversations about? Analysis of vocational training conversations in Dutch vocational education from a career learning perspective. Journal of Vocational Education and Training, 61(3), pp. 247-266. 